\title{
Ruptured aneurysm-induced pituitary apoplexy: illustrative case
}

\author{
Michiharu Yoshida, MD, PhD, ${ }^{1}$ Takeshi Hiu, MD, PhD, ${ }^{1}$ Shiro Baba, MD, PhD, ${ }^{1}$ Minoru Morikawa, MD, PhD, ${ }^{2}$ \\ Nobutaka Horie, MD, PhD, ${ }^{1}$ Kenta Ujifuku, MD, PhD, ${ }^{1}$ Koichi Yoshida, MD, PhD, ${ }^{1}$ Yuki Matsunaga, MD, ${ }^{1}$ Daisuke Niino, MD, PhD, ${ }^{3}$ \\ Ang Xie, BS, ${ }^{1}$ Tsuyoshi Izumo, MD, PhD, ${ }^{1}$ Takeo Anda, MD, PhD, ${ }^{1}$ and Takayuki Matsuo, MD, PhD ${ }^{1}$
}

Departments of ${ }^{1}$ Neurosurgery, ${ }^{2}$ Radiology, and ${ }^{3}$ Pathology, Nagasaki University Graduate School of Biomedical Sciences, Nagasaki, Japan

BACKGROUND Pituitary apoplexy associated with aneurysmal rupture is extremely rare and may be misdiagnosed as primary pituitary adenoma apoplexy. The authors present a case of a patient with pituitary apoplexy caused by rupture of an anterior cerebral artery aneurysm embedded within a giant pituitary adenoma, and they review the relevant literature.

OBSERVATIONS A 78-year-old man experienced sudden headache with progressive vision loss. Magnetic resonance imaging (MRI) revealed a giant pituitary tumor with abnormal signal intensity. Magnetic resonance angiography immediately before surgery showed a right A1 segment aneurysm, suggesting coexisting pituitary apoplexy and ruptured aneurysm. The patient underwent urgent transsphenoidal surgery for pituitary apoplexy. The tumor was partially removed, but the perianeurysmal component was left behind. Subsequent cerebral angiography showed a 5-mm right A1 aneurysm with a bleb that was successfully embolized with coils. Retrospective review of preoperative dynamic MRI showed extravasation of contrast medium from the ruptured aneurysm into the pituitary adenoma. Histopathologic examination showed gonadotroph adenoma with hemorrhagic necrosis. Postoperatively, the patient's visual function improved.

LESSONS MRI identification of pituitary apoplexy caused by aneurysmal rupture has not been reported previously. Aneurysmal rupture should be considered in the differential diagnosis of pituitary apoplexy. When a ruptured aneurysm is encountered, the authors recommend treating it before addressing pituitary apoplexy.

https://thejns.org/doi/abs/10.3171/CASE21169

KEYWORDS anterior cerebral artery aneurysm; nonfunctioning pituitary adenoma; pituitary apoplexy; ruptured aneurysm

Pituitary apoplexy is a clinical syndrome caused by acute hemorrhage or ischemic infarction of the pituitary gland. Pituitary apoplexy associated with a ruptured aneurysm is extremely rare. ${ }^{1}$ We report the first case of pituitary apoplexy caused by rupture of an A1 segment anterior cerebral artery aneurysm that was embedded within a giant nonfunctioning pituitary adenoma, and we review the pathophysiology and relevant literature.

\section{Illustrative Case}

A 78-year-old right-handed man experienced sudden headache and nausea with bitemporal hemianopia and collapsed. On admission to the nearest emergency hospital, brain computed tomography (CT) revealed a high-density mass arising from the sella. Magnetic resonance imaging (MRI) performed 1 day later showed pituitary apoplexy with subarachnoid hemorrhage (SAH).
Three days after symptom onset, the patient was transferred to our hospital because of progressive vision impairment. Neurological examination showed disorientation, right ptosis, bilateral unresponsive pupils, and absence of extraocular movements in all directions. His left eye was blind. In the right eye, light perception was poorly preserved. The finding of endocrinological testing was within normal range.

Repeat imaging studies showed a $5-\mathrm{cm} \times 4-\mathrm{cm} \times 3-\mathrm{cm}$ pituitary macroadenoma with suprasellar extension and right cavernous sinus invasion (Fig. 1A and B). Diffusion-weighted sequences showed high signal within the intrasellar mass. Magnetic resonance angiography immediately before surgery showed a right A1 aneurysm embedded within the tumor. Hematoma was distributed mainly around the aneurysm, suggesting coexisting pituitary apoplexy and ruptured aneurysm. Urgent endoscopic transsphenoidal surgery was performed. The tumor was

ABBREVIATIONS CT = computed tomography; $\mathrm{GH}=$ growth hormone; $\mathrm{MRI}=$ magnetic resonance imaging; PRL = prolactin; $\mathrm{SAH}=$ subarachnoid hemorrhage INCLUDE WHEN CITING Published June 28, 2021; DOI: 10.3171/CASE21169.

SUBMITTED March 27, 2021. ACCEPTED April 7, 2021.

(c) 2021 The authors, CC BY-NC-ND 4.0 (http://creativecommons.org/licenses/by-nc-nd/4.0/). 

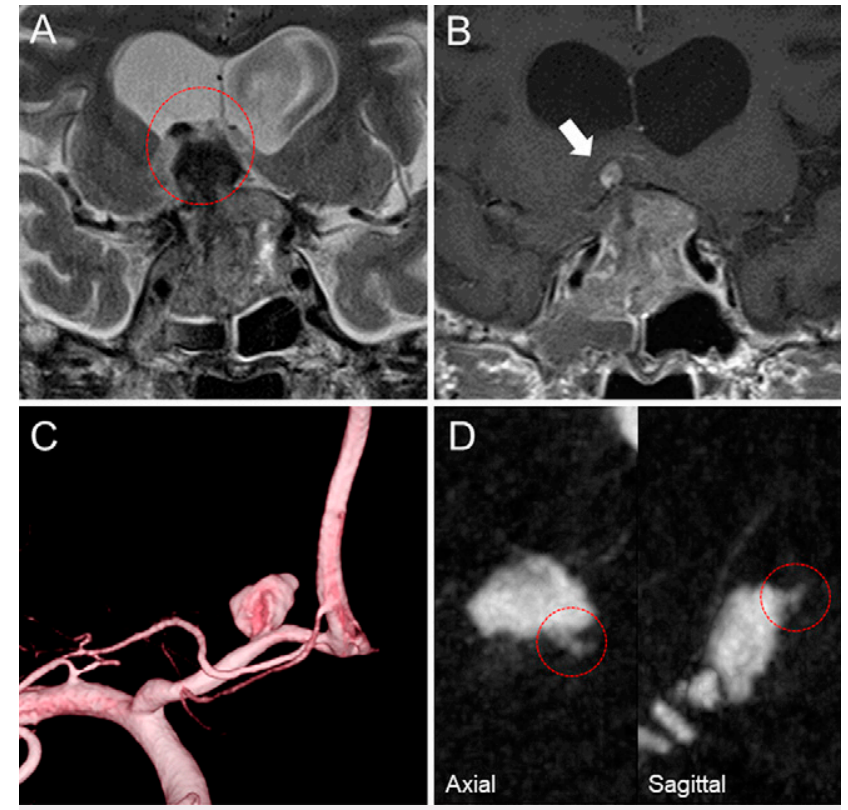

FIG. 1. Coronal contrast-enhanced T1-weighted (A) and coronal T2weighted (B) MRI show abnormal signal intensity within the giant pituitary tumor, right cavernous sinus invasion, and suprasellar extension. The aneurysm (white arrow) was embedded within the tumor. The hematoma (red circle) was distributed mainly around the aneurysm. Three-dimensional (3D) rotational angiography, frontal view (C), shows a right $A 1$ segment aneurysm. Multiplanar $3 \mathrm{D}$ rotational angiography reconstruction (D) demonstrates a superoposteriorly projecting bleb on the aneurysm, which was the presumed rupture point (red circles).

partially removed, and the perianeurysmal component was intentionally left behind. Cerebral angiography on the morning after surgery confirmed an irregular 5-mm right $\mathrm{A} 1$ aneurysm with a superoposteriorly projecting bleb, which was the presumed rupture point (Fig. $1 \mathrm{C}$ and D, and $2 \mathrm{~A}$ ). Complete endovascular occlusion of the aneurysm was then achieved using platinum coils (Fig. 2B). Retrospective review of the preoperative dynamic MRI showed extravasation of contrast medium from the ruptured aneurysm into the pituitary adenoma (Video 1).

VIDEO 1. Clip showing preoperative dynamic MRI depicting extravasation of contrast medium from the ruptured $\mathrm{A} 1$ aneurysm into the pituitary adenoma. Click here to view.

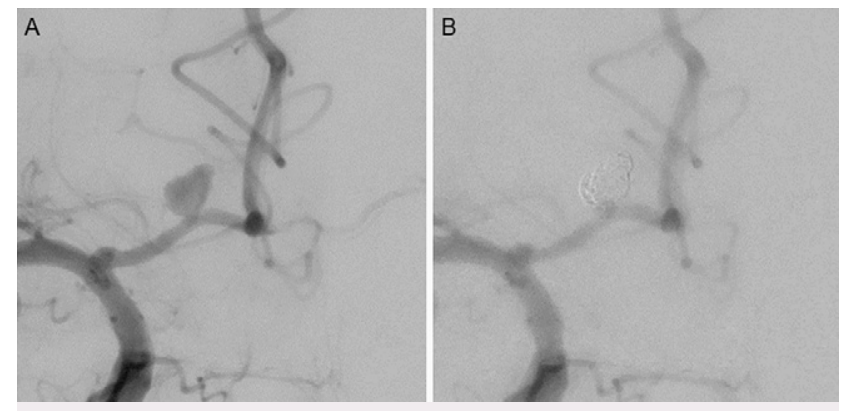

FIG. 2. Digital subtraction angiography, frontal view, shows a right $A 1$ segment aneurysm (A). Complete endovascular occlusion of the aneurysm was achieved (B).

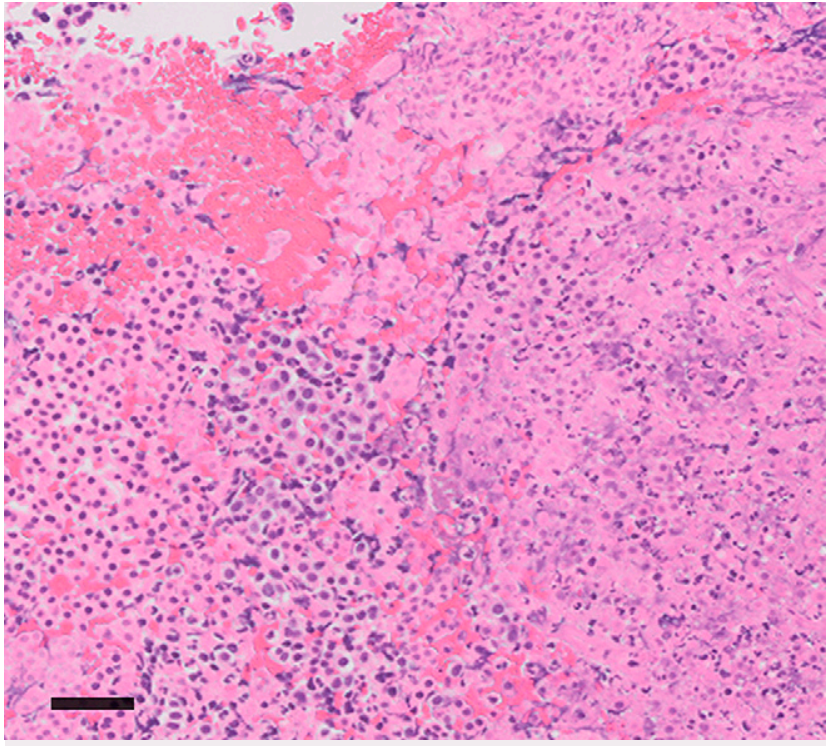

FIG. 3. Histopathology of the pituitary tumor. Hematoxylin and eosin staining demonstrates a hemorrhagic necrosis pattern indicating pituitary apoplexy. Bar $=50 \mu \mathrm{m}$.

Postoperative MRI showed partial tumor removal as expected; the perianeurysmal component remained. Histopathological examination of the surgical tumor specimen revealed both hemorrhagic necrosis (Fig. 3) and typical adenoma on hematoxylin and eosin staining; a typical adenoma was observed over a relatively large area. Immunohistochemical staining was positive for luteinizing hormone and follicle-stimulating hormone, consistent with gonadotroph adenoma. The patient's visual acuity improved to light perception in the right eye and 0.05 in the left.

\section{Discussion}

Observations

Coexisting intracranial aneurysms are found in $2.3 \%$ to $7.4 \%$ of patients with pituitary adenoma, ${ }^{2-4}$ a higher prevalence than that in the general population and in patients harboring other brain tumors. However, pituitary apoplexy associated with intracranial aneurysmal rupture is extremely rare. To our knowledge, only 5 cases have been reported (Table 1). ${ }^{1,5-7}$ The prevalence of coexisting unruptured aneurysm is high in growth hormone (GH)-type pituitary adenomas, ranging from $2.3 \%$ to $13.8 \%$. Conversely, the prevalence is low in prolactin (PRL)-type adenomas $(0.9 \%-2.4 \%),{ }^{2,3,8,9}$ which are occasionally aggressive and invasive. ${ }^{10}$ Two of the 5 reported patients with intracranial ruptured aneurysm associated with pituitary apoplexy harbored PRL-type adenomas, and one harbored a GHtype adenoma; only our case had a nonfunctioning adenoma.

Local circulatory stress, endocrinological effects, mechanical effects, and direct invasion have been proposed as mechanisms underlying aneurysm formation in patients with pituitary adenomas $^{2,11-13}$; however, the contribution of the pituitary adenoma is unclear. ${ }^{2,3}$ Mechanisms other than endocrinological effects may have been involved in our patient with nonfunctioning adenoma.

In a review of cerebral angiograms in 467 pituitary adenoma patients, Pant et al. found aneurysms in 25 (5.4\%), 97\% of which were located in the anterior circulation. ${ }^{3}$ Among the 5 cases of intracranial ruptured aneurysm associated with pituitary apoplexy 
reviewed here, all aneurysms were in the anterior circulation (anterior communicating artery, 2 cases; A1 segment, 2 cases; posterior communicating artery, 1 case). In only 2 cases, including ours, was the ruptured aneurysm embedded within the tumor.

According to Oh et al., older age and cavernous sinus invasion correlate with increased incidence of intracranial aneurysm in patients with pituitary adenomas. ${ }^{2}$ Tumor growth causes microanatomical alterations in the cerebral circulation, blood flow modification, and greater hemodynamic stress, which predispose patients to aneurysm formation. ${ }^{2,8,11,14}$ In our 78-year-old patient, the pituitary tumor invaded the right cavernous sinus, and the right $A 1$ aneurysm was embedded within the tumor. As the tumor gradually grew out of the sella and extended behind the optic chiasm, it may have invaded and weakened the aneurysmal wall, causing rupture.

To the best of our knowledge, MRI identification of pituitary apoplexy caused by rupture of an intracranial aneurysm has not been reported previously. Increased intratumoral pressure at the time of aneurysmal rupture might have caused compression of the pituitary vessels, resulting in secondary pituitary apoplexy.

We performed endoscopic transsphenoidal surgery first because we detected the aneurysm immediately before surgery. Fortunately, our patient experienced improved visual function and avoided fatal aneurysmal rerupture. However, his aneurysm ruptured within the tumor. In general, aneurysm treatment should be given priority when aneurysmal SAH is suspected.

\section{Lessons}

MRI identification of pituitary apoplexy caused by rupture of an intracranial aneurysm has not been reported previously. Aneurysmal rupture should be considered in the differential diagnosis of pituitary apoplexy. When encountered, we recommend treating the ruptured aneurysm before addressing pituitary apoplexy.

\section{Acknowledgments}

We thank Edanz Group for editing a draft of the manuscript.

\section{References}

1. Xu K, Yuan Y, Zhou J, Yu J. Pituitary adenoma apoplexy caused by rupture of an anterior communicating artery aneurysm: case report and literature review. World J Surg Oncol. 2015;13:228.

2. Oh MC, Kim EH, Kim SH. Coexistence of intracranial aneurysm in 800 patients with surgically confirmed pituitary adenoma. J Neurosurg. 2012;116(5):942-947.

3. Pant $B$, Arita K, Kurisu K, et al. Incidence of intracranial aneurysm associated with pituitary adenoma. Neurosurg Rev. 1997;20(1): 13-17.

4. Wakai S, Fukushima T, Furihata T, Sano K. Association of cerebral aneurysm with pituitary adenoma. Surg Neurol. 1979;12(6): 503-507.

5. Laidlaw JD, Tress B, Gonzales MF, et al. Coexistence of aneurysmal subarachnoid haemorrhage and pituitary apoplexy: case report and review of the literature. J Clin Neurosci. 2003;10(4): 478-482.

6. Shahlaie K, Olaya JE, Hartman J, Watson JC. Pituitary apoplexy associated with anterior communicating artery aneurysm and aberrant blood supply. J Clin Neurosci. 2006;13(10): 1057-1062.

7. Song RX, Wang DK, Wang Z, et al. Coexistence of aneurysmal subarachnoid hemorrhage and surgically identified pituitary apoplexy: a case report and review of the literature. J Med Case Rep. 2014;8:166. 
8. Jakubowski J, Kendall B. Coincidental aneurysms with tumours of pituitary origin. J Neurol Neurosurg Psychiatry. 1978;41(11):972-979.

9. Oshino S, Nishino A, Suzuki T, et al. Prevalence of cerebral aneurysm in patients with acromegaly. Pituitary. 2013;16(2):195-201.

10. Shimon I. Giant prolactinomas. Neuroendocrinology. 2019;109(1):51-56.

11. Mangiardi JR, Aleksic SN, Lifshitz M, et al. Coincidental pituitary adenoma and cerebral aneurysm with pathological findings. Surg Neurol. 1983;19(1):38-41.

12. Weir B. Pituitary tumors and aneurysms: case report and review of the literature. Neurosurgery. 1992;30(4):585-591.

13. Choi HS, Kim MS, Jung YJ, Kim OL. Incidental superior hypophygeal artery aneurysm embedded within pituitary adenoma. J Korean Neurosurg Soc. 2013;54(3):250-252.

14. Pia HW, Obrador S, Martin JG. Association of brain tumours and arterial intracranial aneurysms. Acta Neurochir (Wien). 1972;27(3):189-204.

\section{Disclosures}

The authors report no conflict of interest concerning the materials or methods used in this study or the findings specified in this paper.

\section{Author Contributions}

Conception and design: Hiu, Yoshida, Matsuo. Acquisition of data: Hiu, Yoshida, Morikawa, Ujifuku. Analysis and interpretation of data: Hiu, Baba, Morikawa, Anda. Drafting the article: Hiu, Yoshida, Horie. Critically revising the article: Hiu, Yoshida, Baba, Morikawa, Horie, Ujifuku, Yoshida, Matsunaga, Niino, Xie, Izumo. Reviewed submitted version of manuscript: Hiu, Baba, Horie, Ujifuku, Niino. Approved the final version of the manuscript on behalf of all authors: Hiu. Administrative/technical/material support: Morikawa. Study supervision: Hiu.

\section{Supplemental Information Videos}

Video 1. https://vimeo.com/534462897.

\section{Correspondence}

Takeshi Hiu: Nagasaki University Graduate School of Biomedical Sciences, Nagasaki, Japan. thiu.nagasaki@gmail.com. 